\title{
Capecitabine as Maintenance Therapy for High-Risk, Resected Colorectal Cancer
}

\author{
Miklos L. Auber ${ }^{a}$ Sijin Wen ${ }^{b}$ Gerald Hobbs ${ }^{c}$ Gerald M. Higa a,d \\ ${ }^{a}$ Department of Medicine, West Virginia University, Morgantown, WV, USA; bepartment of Biostatistics, West \\ Virginia University, Morgantown, WV, USA; 'Department of Statistics, West Virginia University, Morgantown, WV, \\ USA; ${ }^{d}$ Department of Clinical Pharmacy, West Virginia University, Morgantown, WV, USA
}

\section{Keywords}

Capecitabine · Event-free survival $\cdot$ High-risk colorectal cancer - Maintenance therapy

\begin{abstract}
Introduction: In 2020, colorectal cancer will be the fourth most frequently diagnosed malignant neoplasm and the second leading cause of site-specific, cancer-related deaths in the USA. Notably, $80 \%$ of the new cases are, by staging criteria, potentially curable even those with completely resected stage 4 disease. If slightly more than half the losses can be attributed to metastatic disease at presentation, then the remaining portion of deaths may be linked to disease relapse after surgery and, if applicable, adjuvant chemotherapy. The inference that these therapies are not curative for a significant number of subjects poses a role for maintenance therapy. Objective: To assess event-free survival (EFS) of patients who received capecitabine as maintenance therapy following treatment according to current guidelines. Methods: Clinical outcomes data were collected for 35 subjects treated with capecitabine as maintenance therapy. Descriptive statistical analyses were conducted on collective data related to duration of maintenance therapy and disease or clinical status from surgery to initial event. Kaplan-Meier method and log-rank test were used to analyze EFS and
\end{abstract}

\begin{tabular}{|c|c|}
\hline $\begin{array}{l}\text { karger@karger.com } \\
\text { www.karger.com/gat }\end{array}$ & $\begin{array}{l}\text { (C) } 2021 \text { The Author(s) } \\
\text { Published by S. Karger AG, Basel }\end{array}$ \\
\hline Karger $\frac{V}{\%}$ & $\begin{array}{l}\text { This article is licensed under the Creative Commons Attribution- } \\
\text { NonCommercial-NoDerivatives } 4.0 \text { International License (CC BY- } \\
\text { NC-ND) (http://www.karger.com/Services/OpenAccessLicense). } \\
\text { Usage and distribution for commercial purposes as well as any dis- } \\
\text { tribution of modified material requires written permission. }\end{array}$ \\
\hline
\end{tabular}

overall survival. Results: Of the entire cohort, 26 subjects have no evidence of disease (NED), a median of 5.5 years from surgery. Kaplan-Meier analyses indicated a 5-year EFS rate of $74 \%$ ( $95 \% \mathrm{Cl}: 60-90 \%)$. Eighteen of these 26 patients received capecitabine $\geq 30$ months. Eight of the 17 subjects treated with capecitabine therapy for $<30$ months developed progressive disease; the majority of the relapses occurred within 20 months of surgery. The difference between the two groups was statistically significant. Six subjects died, only two of who had metastatic disease at the time of death; the other four had NED at least 4 years from surgery. Five patients with resected stage 4 disease who received capecitabine as maintenance therapy were alive $>5$ years from surgery. Conclusion: The findings and analyses of this cohort of patients suggest that maintenance capecitabine therapy reduces the risk of disease progression and cancerrelated death.

(c) 2021 The Author(s)

Published by S. Karger AG, Basel

\section{Introduction}

An estimated 334,000 malignant tumors of the digestive system will be diagnosed in the USA in 2020 [1]. Although this figure represents only $18 \%$ of all new cancer diagnoses, this percentage is still higher than any other 
system-related cancer. Further assessment of these cases indicates that site-specific colorectal cancers (CRCs) account for nearly one-half and one-third of all the digestive system tumors and deaths, respectively.

Based on staging criteria alone, the 5-year relative survival rates for stages 1 and $2 \mathrm{~A}$ colon cancer are $93 \%$ and $72 \%$, respectively; the 5 -year survival rate for stage $3 \mathrm{~A}$ with systemic adjuvant therapy is $73 \%$. However, these survival figures are not observed among all subsets of stage 2 and 3 tumors. While improved survival is usually associated with early stage, one anomaly exists with stages $2 \mathrm{~B}$ and $2 \mathrm{C}$ where the 5 -year survival rates have been reported to be $51.6 \%$ and $32 \%$, respectively [2]. Similarly, the survival figures are $45 \%$ and $33 \%$ for patients with stage $3 \mathrm{~B}$ and $3 \mathrm{C}$ disease, respectively.

Since surgery alone had been the standard of care for stage 2 colon cancer, it is conceivable that a consequential number of patients relapsed and died because of distant disease. With the introduction of molecular testing for genetic and/or gene-encoded protein aberrations, adjuvant chemotherapy may also be considered in subjects with stage $2 \mathrm{~B}$ or $2 \mathrm{C}$ disease as well as stage $2 \mathrm{~A}$ [3]. Furthermore, a small, but potentially significant, proportion of patients with stage $3 \mathrm{~B}$ and $3 \mathrm{C}$ disease will not be cured with surgery plus standard adjuvant chemotherapy.

Recent estimates indicate 150,000 new cases and 50,000 CRC-related deaths in 2020 [1]. Inherent, but not apparent, in these figures is the observation that approximately $80 \%$ of the new diagnoses are classified as stages 1-3 or resectable stage 4 and, therefore, potentially curable. If slightly more than half the loss is attributable to metastatic disease at presentation, then the remaining portion of deaths may be linked to disease relapse after surgery and, if applicable, adjuvant chemotherapy. The inference that these therapies are not curative for a significant number of subjects poses a role for maintenance therapy.

Although clinical trials of maintenance therapy have been conducted with variable results, it is important to emphasize that these trials included only patients with metastatic colon cancer [4-9]. The inconsistent findings of previous reports are likely related to a number of factors including differences in tumor biology as well as the therapeutic agent(s) used. However, tumor burden, at the time maintenance therapy was initiated, may partially account for the disparate results. Because of the latter possibility, it is conceivable that application of maintenance therapy could have clinical benefit in early-stage, high-risk disease. And, considering the elements of cost, route of administration, side effect profile, and quality of life, an oral fluoropyrimidine prodrug could be an appropriate treatment option. The primary aim of this paper is to report preliminary results related to the use of capecitabine as maintenance therapy after standard treatment in a cohort of patients with high-risk, surgically resected CRC.

\section{Patients and Methods}

The majority of patients included in this report had, at the time of diagnosis, pathologically staged $2 \mathrm{C}, 3 \mathrm{~A}, 3 \mathrm{~B}$, or $3 \mathrm{C}$ cancer involving the colon or rectum. All subjects in these subsets with colon cancer underwent surgery followed by standard adjuvant chemotherapy; patients with rectal cancer received neoadjuvant chemoradiation therapy followed by surgery and adjuvant chemotherapy. Five additional patients were diagnosed with stage 4 tumors, 2 of who had extrahepatic metastases. The 3 subjects with liver metastases received neoadjuvant radiofrequency ablation (RFA); and one of these patients was also treated with intrahepatic arterial (IHA) infusion of floxuridine (FUDR). All 5 subjects had no measurable disease following RFA +/- FUDR as mentioned, neoadjuvant chemotherapy, surgery, and systemic adjuvant therapy.

All of the subjects were informed that their disease had characteristics considered high risk for relapse and that the option of further chemotherapy was not considered standard of care. Still, all of the subjects in this report expressed that they wanted to try anything that could possibly prevent disease relapse. In order to fulfill this desire, patients were provided with a description of the proposed intervention, the importance of the individual's role in decision-making, a summary of the standard alternative, and a review of risks associated with the proposed intervention; possible reduction in risk of relapse was mentioned but not magnified. All of these discussion points form the basis of respect for persons, beneficence, and justice taken from the Belmont Report [10]. All patients gave verbal informed consent (which was witnessed by G.M.H. in addition to M.L.A., and documented in their medical records) to be treated with capecitabine as maintenance therapy with the understanding that treatment would be continued till disease progression, intolerable toxicity, or patient or physician decision to stop, whichever came first.

\section{Study Design}

Following adjuvant therapy, the prescribed treatment dose of capecitabine was $1,500-2,000 \mathrm{mg} / \mathrm{m}^{2} /$ day in divided doses for 1 week, every other week. A priori adjustments to this dosage were based on patient age, comorbidities, and performance status. Kidney function did not influence drug dose though patients with calculated creatinine clearances $<30 \mathrm{~mL} / \mathrm{min}$ were not involved; body surface area was capped at $2 \mathrm{~m}^{2}$. The planned course of maintenance therapy was 60 months with the stipulation related to stopping mentioned previously. The primary objective of this report was to assess event-free survival (EFS) of patients who received capecitabine as maintenance therapy.

\section{Statistical Analyses}

Descriptive statistical analyses were performed on the entire cohort to summarize data related to time from surgery to last as-
Auber/Wen/Hobbs/Higa 
Table 1. Composite data of subjects treated with maintenance capecitabine

\begin{tabular}{lc}
\hline Variables & $N(\%)$ \\
\hline Gender & \\
F & $12(34.3)$ \\
M & $23(65.7)$ \\
Stage (in detail) & \\
2C & $2(5.7)$ \\
3A & $2(5.7)$ \\
3B & $14(40)$ \\
3C & $12(34.3)$ \\
4 & $5(14.3)$ \\
Disease status at time of data collection & $26(74.3)$ \\
NED & $3(8.6)$ \\
Stable & $6(17.1)$ \\
Metastatic & Median $($ range $)$ \\
\hline & $64(40,87)$ \\
\hline Age & $0.33(0.13,0.73)$ \\
SEER 5-year survival rate & $30(3,60)$ \\
Duration of capecitabine, months & $5.5(1.33,17.8)$ \\
Time from surgery to last assessment, years & \\
\hline
\end{tabular}

NED, no evidence of disease.

sessment, disease status at the time of this report, and EFS, including summary tables, waterfall plot, median, and standard deviations. Kaplan-Meier method and log-rank test were used to analyze EFS and overall survival.

\section{Results}

Thirty-five subjects included in this report were, or continue to be, treated with capecitabine as maintenance therapy. Numbers of colon and rectal cancer cases were 20 and 15, respectively. A summary of key data is shown in Table 1.

Duration of follow-up is shown in Figure 1. Of the entire cohort, 26 subjects have no evidence of disease (NED) when last seen in the clinic, a median of 5.5 years from surgery. The Kaplan-Meier method indicated a 5-year EFS of 74\% (95\% CI: 60-90\%) (Fig. 2). Eighteen of these 26 patients received capecitabine $\geq 30$ months. In comparison, 8 of the 17 subjects who received maintenance capecitabine therapy for less than the median duration (i.e., 30 months) developed progressive disease; the majority of the relapses occurred within 20 months of surgery. Overall, when all 35 patients were dichotomized by the median duration of capecitabine therapy, the differ-

Maintenance Capecitabine in Resected CRC

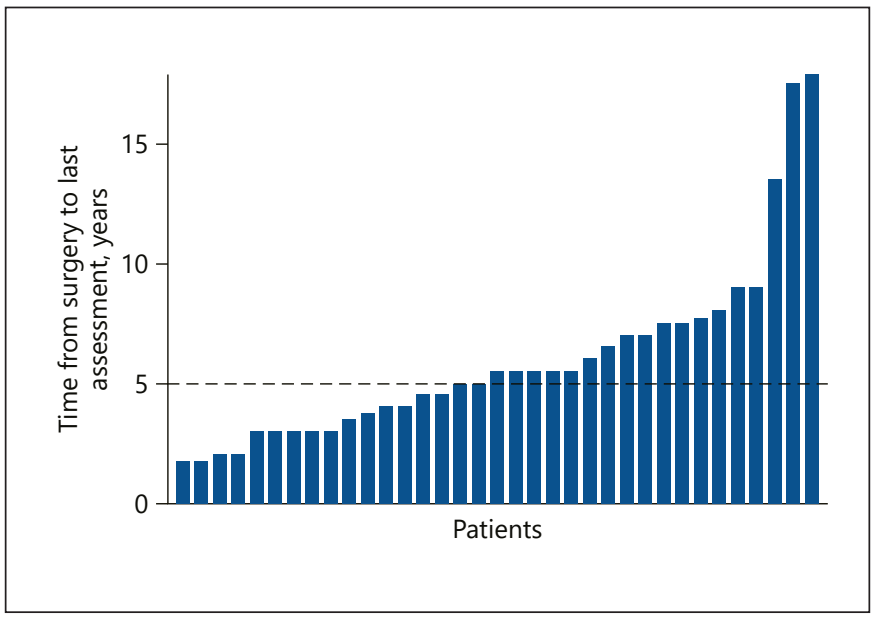

Fig. 1. Waterfall plot of time from surgery to last assessment (median 5.5 years).

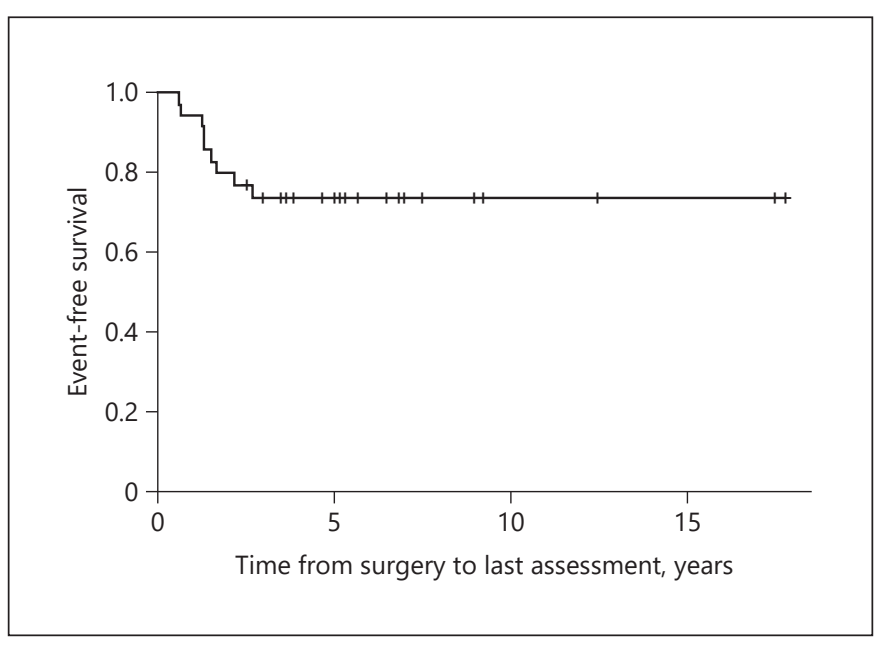

Fig. 2. Kaplan-Meier plot of EFS, 74\% (95\% CI: 60-90\%). EFS, event-free survival.

ence of EFS between the two groups was statistically significant (Fig. 3). In particular, the 5-year EFS is $100 \%$ in patients with capecitabine duration $\geq 30$ months compared to $43 \%$ in patients with capecitabine duration $<30$ months. In addition, the Kaplan-Meier estimate of the 5-year overall survival rate was 78\% (95\% CI: 63-96\%).

A total of six subjects died, only two of who had metastatic disease at the time of death. The other four had NED at least 4 years from surgery. Five patients with stage 4 disease at diagnosis received capecitabine as maintenance therapy following neoadjuvant induction chemotherapy, surgery, and systemic adjuvant therapy; all 5 are 


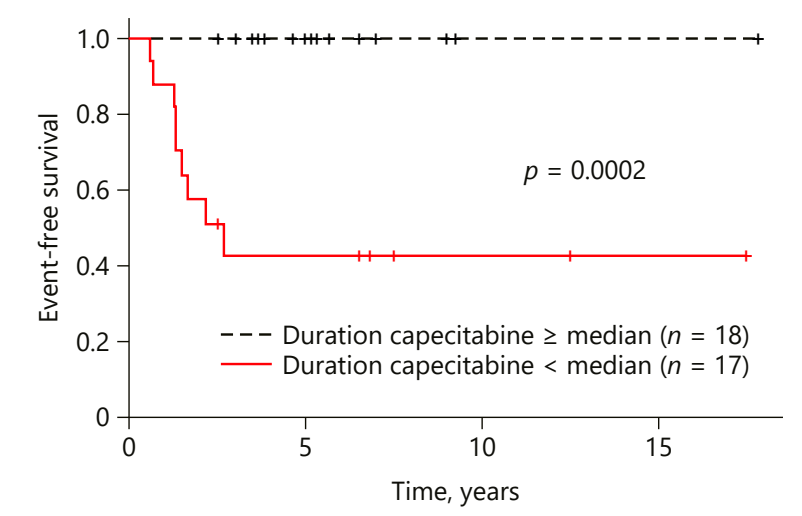

Fig. 3. Kaplan-Meier plot of EFS by duration of capecitabine therapy (median 30 months, $p$ value $=0.0002$ from the log-rank test). EFS, event-free survival.

alive $>5$ years from surgery. Four of the subjects have been on maintenance capecitabine for $>4$ years with NED.

The most common side effect observed was grade $1 / 2$ hand-foot syndrome which occurred in nearly all of the subjects. The relatively low-grade toxicity responded to, or resolved with, dose reduction (500 mg of the total daily dose).

\section{Discussion}

Notwithstanding the availability of screening tests, the mortality rate of CRC is still more than twice that of breast cancer in their respective incident populations. Part of the reason for the latter observation is related to the absence of key predictive markers to guide therapy. Even $K-R A S$ appears to be, at best, only marginally important because it may not be a major tumor driver. Indeed, response to cetuximab or panitumumab treatment is observed in $<10 \%$ of patients with metastatic CRC (mCRC) who exhibit wild-type $K-R A S$, and disease progression will occur in the majority of these patients $[11,12]$.

It has been clearly shown that addition of adjuvant systemic therapy has improved disease-free and overall survival in patients with operable breast and CRCs [13-16]. One striking difference between the two tumor types relates to the duration of adjuvant therapy, particularly in patients with hormone receptor-positive, HER2-negative early breast cancer. Furthermore, patient age, anatomic stage, tumor size, lymphovascular invasion, extent of nodal involvement, tumor grade, and genomic signature are used to identify women with ER-positive tumors, some of who will benefit from addition of chemotherapy. In addition to these considerations, obstruction of the lumen and genetic instability have been associated with relapse risk among patients diagnosed with early-stage CRC $[17,18]$.

Data in this report appear to support the belief that some patients will gain additional benefit by extending the duration of systemic therapy beyond 6 months (of adjuvant therapy). Of particular interest is the median 5 -year EFS rate of 74\% for the entire group of patients reported herein. This relatively good outcome is consistent with the SEER Database estimate for patients with stage $3 \mathrm{~A}$ disease; only 2 subjects in this study's cohort had stage $3 \mathrm{~A}$ tumors at diagnosis.

Equally noteworthy were the five patients with mCRC at diagnosis who received maintenance capecitabine following RFA with or without IHA infusion of FUDR (in those with liver metastases) and induction FOLFOX followed by surgery and adjuvant chemotherapy. Despite the small number, it is important to emphasize that median overall survival of patients with mCRC is approximately 30 months [19]. That all 5 are alive, 4 of who have NED for $>5$ years from surgery suggest that this finding may be partially attributable to maintenance therapy.

Although a formal quality of life tool was not utilized, none of the subjects developed side effects that necessitated treatment discontinuation. One other concern of maintenance therapy relates to nascent bone marrow abnormalities. A single institution reported a small number of patients treated for CRC who were subsequently diagnosed with acute leukemia or myelodysplasia [20]. However, limited data precluded any statement regarding a causal relationship between prior therapy and incident hematologic malignancies. Instead, the authors speculated that incorporation of oxaliplatin, rather than fluorouracil, may be the link between anticancer therapy and latent marrow aberrances. Adding further credence to their notion regarding the lack of culpability of the fluoropyrimidine is the absence of any reported premalignant marrow findings or leukemogenic effect associated with capecitabine therapy.

When assessing the significance of clinical data, especially findings that were retrospectively collected, there is an inherent obligation to address study limitations. First, using medical records to source data is frequently associated with the inability to assess all facets of patient outcomes. However, all subjects in this report were patients of only one oncologist. As such, the accuracy of the data 
collected is supported by meticulous recording of histories related to date of and stage at diagnosis, all anticancer treatments received by the patients, and detailed notes on follow-up visits. Despite the diligent record keeping, some information was not retrievable such as the cause of death of 4 subjects. However, all of these patients had been followed for $>3$ years and when last seen, none had any evidence of recurrent disease. Second, data collected included only patients who (a) were deemed to be high risk for relapse at diagnosis and (b) provided verbal consent to be treated with capecitabine as maintenance therapy. While these subjects were "selected" according to the above criteria, the findings were not biased by selection based primarily on "best" individual outcomes. Third, the duration of follow-up for half of the entire population was $<5$ years from surgical resection, typically an event-free endpoint which approximates cure. However, all patients in this particular subset were followed over 2 years following surgery, an important figure because the majority of the subjects with disease progression relapsed within 24 months of tumor resection. Fourth, the dose of capecitabine used was lower than the FDA-approved dose. A priori attenuation of the dosage was justified based on clinical experience that few subjects have been able to tolerate the recommended starting dose of 2,500 $\mathrm{mg} / \mathrm{m}^{2} /$ day. Furthermore, a dose-response relationship has not been established for capecitabine; and lower doses may be associated with a better therapeutic index [21]. Fifth, the small sample size precludes making any statement regarding therapeutic effectiveness as statistical inference is based on the univariate data analysis without adjusting for treatment factor. Even so, these early findings provide support that maintenance therapy could improve disease outcomes and justify a randomized clinical trial. Sixth, lack of randomization to a no-treatment arm challenges proscribing a definite conclusion. Nonetheless, the data were compared to estimates of survival generated by SEER which is frequently used by clinicians for prognostic purposes.

\section{Conclusion}

Capecitabine is approved for the treatment of patients with mCRC. This indication plausibly suggests that the drug could be more efficacious when applied in the earlystage, low-tumor burden disease setting. Heretofore, proofof-concept maintenance therapy has never been tested in patients with surgically resected, high-risk CRC. The initial findings of this retrospective analysis suggest that some pa- tients, especially those with stage $2 \mathrm{C}$ (and likely 2B), 3B, 3C, or completely resected stage 4 disease, will gain additional benefit by extending the duration of systemic therapy. While the use of capecitabine beyond six months (of standard adjuvant therapy, usually the FOLFOX regimen) is practicable the optimal dose, schedule and duration of maintenance therapy remain undetermined.

\section{Acknowledgement}

S.W. was supported in part by the US National Institute of Health (NIH) grants U54 GM104942. The content is solely the responsibility of the authors and does not necessarily represent the official views of the NIH.

\section{Statement of Ethics}

Retrospective collection of clinical outcomes data for analysis received full approval of the Institutional Review Board of West Virginia University (IRB Protocol Notice: Protocol 2009110099 Approved). Relevant patient-specific data were accessed from electronic medical records (Epic Hyperspace ${ }^{\mathrm{R}}$ ). Since this was not a formal clinical trial, written informed consent forms were not mandated. Still, all subjects in this report gave fully informed consent for treatment as indicated in the Patients and Methods section.

\section{Conflict of Interest Statement}

The authors do not have any relationship, financial or otherwise (i.e., support in the form of employment, consultancies, honoraria, stock ownership and options, expert testimony, grants or patents received or pending, or royalties), with the manufacturer of the agent described that influenced the writing of this manuscript.

\section{Author Contributions}

M.L.A. contributed to study concept, data acquisition, and manuscript review and editing. S.W. contributed to data analyses and manuscript review. G.H. contributed to data analyses and manuscript review. G.M.H. contributed to study concept, data acquisition and interpretation, and presided over writing and editing the manuscript. The authors approved the final draft of the manuscript and are accountable for all aspects of the work including its accuracy and integrity and all parts of their involvement in this proffered article.

References

1 Siegel RL, Miller KD, Jemal A. Cancer statistics, 2017. CA Cancer J Clin. 2020;67(1):7-30.

2 Amin MB, Edge S, Greene F, Byrd DR, Brookland RK, Washington MK, et al. AJCC cancer staging manual. 8th ed. New York, NY: Springer; 2017. p. 252-4

3 Benson III AB, Venook AP, Cederquist L, Chan E, Chen Y-J, Cooper HS, et al. Colon cancer, version 1.2017. J Natl Compr Canc Netw. 2017;15:370-98. 
4 Aparicio T, Ghiringhelli F, Boige V, 1 Malicot $\mathrm{K}$, Taieb J. Bouché $\mathrm{O}$ for the PRODIGE 9 Investigators. Bevacizumab maintenance versus no maintenance during chemotherapy-free intervals in metastatic colorectal cancer: a randomized phase III trial (PRODIGE 9). J Clin Oncol. 2018;36:674-81.

5 Goey KKH, Elias SG, van Tinteren H, Laclé MM, Willems SM, Offerhaus GJA, et al. Maintenance treatment with capecitabine and bevacizumab versus observation in metastatic colorectal cancer: updated results and molecular subgroup analyses of the phase 3 CAIRO3 study. Ann Oncol. 2017;28(9):212834.

6 Luo HY, Li YH, Wang W, Wang ZQ, Yuan X, $\mathrm{Ma} D$, et al. Single-agent capecitabine as maintenance therapy after induction of XELOX (or FOLFOX) in first-line treatment of metastatic colorectal cancer: randomized clinical trial of efficacy and safety. Ann Oncol. 2016;27(6):1074-81.

7 Hegewisch-Becker S, Graeven U, Lerchenmüller CA, Killing B, Depenbusch R, Steffens $\mathrm{CC}$, et al. Maintenance strategies after firstline oxaliplatin plus fluoropyrimidine plus bevacizumab for patients with metastatic colorectal cancer (AIO 0207): a randomised, non-inferiority, open-label, phase 3 trial. Lancet Oncol. 2015;16(13):1355-69.

8 Simkens LH, van Tinteren H, May A, ten Tije AJ, Creemers GJ, Loosveld OJ, et al. Maintenance treatment with capecitabine and bevacizumab in metastatic colorectal cancer (CAIRO3): a phase 3 randomised controlled trial of the Dutch Colorectal Cancer Group. Lancet. 2015;385(9980):1843-52.
9 de Souza JA, Polite B, Perkins M, Meropol NJ, Ratain MJ, Newcomer LN, et al. Unsupported off-label chemotherapy in metastatic colon cancer. BMC Health Serv Res. 2012;12(12): 481.

10 Department of Health, Education, and Welfare. Ethical principles and guidelines for the protection of human subjects of research, report of the national commission for the protection of human subjects of biomedical and behavioral research. Fed Reg. 1979;44:231927.

11 Cunningham D, Humblet Y, Siena S, Khayat D, Bleiberg H, Santoro A, et al. Cetuximab monotherapy and cetuximab plus irinotecan in irinotecan-refractory metastatic colorectal cancer. N Engl J Med. 2004;351(4):337-45.

12 Van Cutsem E, Peeters M, Siena S, Humblet Y, Hendlisz A, Neyns B, et al. Open-label phase III trial of panitumumab plus best supportive care compared with best supportive care alone in patients with chemotherapy-refractory metastatic colorectal cancer. J Clin Oncol. 2007;25(13):1658-64.

13 Breast International Group (BIG) 1-98 Collaborative Group; Thürlimann B, Keshaviah A, Coates AS, Mouridsen H, Mauriac L, et al. A comparison of letrozole and tamoxifen in postmenopausal women with early breast cancer. N Engl J Med. 2005;353(26):2747-57.

14 Pan H, Gray R, Braybrooke J, Davies C, Taylor C, McGale P, et al. EBCTCG. 20-year risks of breast-cancer recurrence after stopping endocrine therapy at 5 years. N Engl J Med. 2017; 377:1836-46.
15 Shah MA, Renfro LA, Allegra CJ, André T, de Gramont A, Schmoll HJ, et al. Impact of patient factors on recurrence risk and time dependency of oxaliplatin benefit in patients with colon cancer: analysis from modern-era adjuvant studies in the adjuvant colon cancer end points (ACCENT) database. J Clin Oncol. 2016;34(8):843-53.

16 Sargent D, Sobrero A, Grothey A, O'Connell MJ, Buyse M, Andre T, et al. Evidence for cure by adjuvant therapy in colon cancer: observations based on individual patient data from 20,898 patients on 18 randomized trials. J Clin Oncol. 2009;27(6):872-7.

17 Kim YH, Petko Z, Dzieciatkowski S, Lin L, Ghiassi M, Stain S, et al. CpG island methylation of genes accumulates during the adenoma progression step of the multistep pathogenesis of colorectal cancer. Genes Chromosomes Cancer. 2006;45(8):781-9.

18 Ulanja MB, Rishi M, Beutler BD, Sharma M, Patterson DP, Gullapalli N, et al. Colon cancer sidedness, presentation, and survival at different stages. J Oncol. 2019;21:4315032.

19 Van Cutsem E, Cervantes A, Adam R, Sobrero A, van Krieken JH, Aderka D, et al. ESMO consensus guidelines for the management of patients with metastatic colorectal cancer. Ann Oncol. 2016;27(8):1386-422.

20 Stein EM, Pareek V, Kudlowitz D, Douer D, Tallman MS. Acute leukemias following a diagnosis of colorectal cancer: Are they therapy-related? Blood. 2012;120(21):1453.

21 Hennessy BT, Gauthier AM, Michaud LB, Hortobagyi G, Valero V. Lower dose capecitabine has a more favorable therapeutic index in metastatic breast cancer: retrospective analysis of patients treated at M. D. Anderson Cancer Center and a review of capecitabine toxicity in the literature. Ann Oncol. 2005;16(8):1289-96. 